\title{
A Pilot Evaluation of Magnetic Resonance Imaging Characteristics Seen With Primary Soft Tissue Giant Cell Tumor in 2 patients
}

\section{Lina Zhang ( $\nabla$ zln201045@163.com )}

First Affiliated Hospital of Dalian Medical University https://orcid.org/0000-0003-2242-9249

Jianyun Kang

First Affiliated Hospital of Dalian Medical University

Kai Zhang

Second affiliated hospital of Dalian Medical University

Ailian Liu

First Affiliated Hospital of Dalian Medical University

Huali Wang

First Affiliated Hospital of Dalian Medical University

Tieli Liu

Dalian Medical University

Research article

Keywords: Soft tissue giant cell tumor, Magnetic resonance imaging, Diffusion weighted imaging

Posted Date: September 30th, 2019

DOI: https://doi.org/10.21203/rs.2.15411/v1

License: (9) (i) This work is licensed under a Creative Commons Attribution 4.0 International License.

Read Full License 


\section{Abstract}

Background Primary soft tissue giant cell tumor (GCT-ST) is a rare tumor with low malignant potential. Here we reported two cases of patients with soft tissue giant cell tumor in the limb, including their clinical and imaging findings (conventional Magnetic resonance imaging (MRI) and Diffusion weighted imaging (DWI)).Methods This retrospective study included two pathology-confirmed GCT-ST patients. Plain MRI, dynamic contrast enhancement MRI (DCE-MRI), and DWI were performed with a 3.0T whole-body MR scanner before surgery. The following characteristics of lesion were recorded: signal intensity on $\mathrm{T} 1$ FSPGR and T 2 WI, morphology, maximum lesion size, time intensity curve (TIC) on DCE-MRI, and apparent diffusion coefficient (ADC) value from DWI.Results The maximum lesion size ranged from 4.0 $\mathrm{cm}$ to $6.0 \mathrm{~cm}$. Signal intensities of all lesions were heterogeneous on T 1 FSPGR and T 2 WI. Nodular enhancements were observed for all lesions with either oval or irregular shapes on MRI. All lesion margins were blurred, and internal enhancements were heterogeneous on DCE-MRI. TIC appeared with a slow increase type. Lesions on DWI ( $b=500 \mathrm{~s} / \mathrm{mm} 2)$ were hyperintense with an higher mean ADC value of $2.19 \times 10-3 \mathrm{~mm} 2$ /s compared to surrounding normal soft tissue $(1.03 \times 10-3 \mathrm{~mm} 2 / \mathrm{s})$.Conclusions DWI may be a useful tool for differentiating benign soft tissue mass from giant cell-rich soft tissue neoplasms or malignant tumors.

\section{Background}

Primary giant cell tumor of soft tissue (GCT-ST) is a rare tumor with low malignant potential that is located in superficial and deep soft tissues ${ }^{[1]}$. Histologically, GCT-ST lesions bear a close resemblance to their bony counterparts, giant cell tumor of bone. Most of the existing reports are pathological and clinical studies, while the imaging aspects have only been reported in the cases of adjacent bone or with atypical cystic degeneration ${ }^{[2-10]}$. There is only one case report on intravoxel incoherent motion (IVIM) DWI of juxtacortical mass ${ }^{[2]}$. Therefore, this study aims to fill the gap in the current MRI knowledge of GCT-ST of the limb by using comprehensive medical imaging examinations (especially conventional MRI added with quantitative DWI).

\section{Methods}

\section{Patient selection}

Two GCT-ST patients who were treated at our center between August 2018 and February 2019 were included in this study. Both patients were diagnosed with GCT-ST through pathology after complete surgical excision and had a unilateral lesion located in the muscle of the limb.This study was approved by the ethics committee of First Affiliated Hospital of Dalian Medical University (Dalian, China). The written informed consent was obtained from all participants.

\section{MR Imaging}


All MR examinations were performed on a 1.5T whole-body MR scanner (Signa, Excite, HDx, General Electric Healthcare, Milwaukee, WI). The MRI protocols were as follows: 1) conventional MR scan sequences included Coronal or axial Fast spin-echo (FSE) $T_{2}$-weighted images, $T_{1}$-weighted images and $T_{1}$ fat-saturation spoiled gradient recalled echo (FSPGR). 2) Axial dynamic 3D $T_{1} F S P G R$ (Lava) sequence (total 4 phases, $T R / T E=6.0 / 3.0 \mathrm{~ms}, F A=12^{\circ}$, slice thickness $/$ slice spacing $=5.0 \mathrm{~mm} / 2.5 \mathrm{~mm}$, acquisition time $=23 \mathrm{~s}$ (one phase) was performed after injecting gadolinium using a antecubital vein power injector at a rate of $2.0 \mathrm{ml} / \mathrm{s}$ with a dose of $0.1 \mathrm{mmol} / \mathrm{kg}$ followed by $20 \mathrm{ml}$ saline; the first acquisition started 25 seconds after contrast agent injection. 3) DWI $\left(b=500 \mathrm{~s} / \mathrm{mm}^{2}\right)$ with repetition time/echo time (TR/TE) of $4700 \mathrm{~ms} / 69 \mathrm{~ms}$, slice thickness/slice spacing of $6.0 \mathrm{~mm} / 1.5 \mathrm{~mm}$, field of view of $288 \mathrm{~mm}$ and reconstruction matrix size of $256 \times 256$ were used; DWI was performed before contrast injection.

Adding qualitative and quantitative data to conventional MRI can improve the diagnosis of benign and malignant soft-tissue masses ${ }^{[11]}$. In this study, DWI was added to our protocol to investigate the performance of DWI in GCT-ST diagnosis.

\section{Image analysis}

All images were transferred to a GE workstation (Advantage Windows 4.5; General Electric, Madison, WI, USA) for image processing and were interpreted by two radiologists with more than five years of diagnostic experience. Consensus was reached for situations in which there was a diagnostic discrepancy between the two readers, which was used in the final MRI analysis. The following lesion characteristics were recorded: 1 ) signal intensity on $T_{2} W I$ and $\left.T_{1} F S P G R, 2\right)$ morphology and maximum lesion size on dynamic contrast enhancement MRI (DCE-MRI), 3) time intensity curve (TIC) from DCE-MRI, 4) apparent diffusion coefficient (ADC) value from DWI.

\section{Results}

Clinical findings included patient age, location, physical examination, and patient history. In case 1, a 60year-old man was referred to our department for evaluation of a painless, slow-growing right-sided mass on the thigh next to the inguinal flexure. Physical examination revealed a $4.0 \times 2.0 \mathrm{~cm}$ firm, poorly circumscribed and non-movable mass. There was no history of fever, injury and skin redness. Laboratory data, including calcium, phosphate and alkaline phosphatese values, were all within the normal ranges.

In case 2, a 36-year-old woman was admitted to our hospital with swelling, skin redness and pain in the upper limb that persisted for 6 months without prior history of trauma. A clinical examination revealed that the $6.0 \times 4.0 \mathrm{~cm}$ mass lesion was tender and did not display any discharge or drainage sinus. No lymph node metastasis was found in any case, and both cases showed no evidence of metastasis or recurrence at half to one-year follow up.

Both patients underwent US and CT scan. For case 1, US revealed a well-circumscribed large solid mass located in the gracilis muscle. The Doppler sonographic examination showed a few vascular signals 
within the mass (Fig. 1a). Plain CT showed an isodensity mass with enlargement of gracilis muscle, while contrast CT revealed a slightly persistent homogeneous enhancement of the mass with blurred margins (Fig. 1b-d). For case 2, US revealed a blur large solid mass located in the deltoid muscle. Plain CT showed a hypodensity mass located in the superficial deltoid muscle extending to the intermuscular space, while contrast CT revealed a slightly persistent heterogeneous enhancement of the mass with blurred margins (Fig. 1ef). There were no evidences of calcification or mineralization in any of the mass, and the adjacent structures were all normal.

On MRI, the morphological manifestations of the masses were showed intuitively. The maximum lesion size ranged from $4.0 \mathrm{~cm}$ to $6.0 \mathrm{~cm}$. All lesions showed iso-hyper signal intensities on $\mathrm{T}_{2} \mathrm{WI}$ with fat saturation (Fig. 2a, white arrow) or without fat saturation (Fig. 3a white arrow) sequence, and heterogeneous hypo-iso signal intensities on $\mathrm{T}_{1}$ FSPGR sequence (Fig. 2b, white arrow; Fig. 3b white arrow), relative to muscle signal intensity. The solid masses showed well-defined margins adjacent to the subcutaneous tissue, while the blurred margins adjacent to the muscle showed a slight hyperintense signal on $\mathrm{T}_{2}$-weighted images (Fig. 2c, black triangle). On DCE-MRI, all lesions showed single or multiple nodular enhancements with either oval (Fig. 2d white arrow) or irregular (Fig. 3c) shapes, and with blurred margins. Furthermore, internal enhancements were all heterogeneous. TIC appeared with a slow increase type (Fig. 3d). Lesions on DWI ( $b=500 \mathrm{~s} / \mathrm{mm}^{2}$ ) were hyperintense with an higher mean ADC value of $2.19 \times$ $10^{-3} \mathrm{~mm}^{2} / \mathrm{s}$ (Fig. 3e, ROI 3) than surrounding normal soft tissue $\left(1.03 \times 10^{-3} \mathrm{~mm}^{2} / \mathrm{s}\right)$ (Fig. 3f, ROI2).

The resected specimens revealed that the tumors invaded the peripheral muscles. Consequently, complete surgical excision of the mass was performed. Pathological examination indicated that many multinucleated giant cells were scattered, and were surrounded by spindle cells, which suggested a GCTST. The giant and spindle cells had slight cellular atypia. In addition, spindle cells exhibited brisk mitotic activity. The tumor invaded the neighboring muscles in both cases. The obtained data suggested that this was a slow-growing entity.

Tumors interstitial hemorrhage were obvious and rich in hemosiderin-containing cells (Fig. 4 a-d). Immunohistochemically, the giant cells were strongly positive for CD68 in both cases. In addition, compared to Case 2, smooth-muscle actin (SMA) of Case 1 showed focally positive staining. In addition, no staining of Myoglobin, Myogenin, MyoD1, desmin, CD34, S-100, CK and EMA were found in both tumors (Fig. $4 \mathrm{e}-\mathrm{f}$ ). Finally, based on clinical, histologic and immunohistochemical (IHC) findings, patients were diagnosed with giant cell tumors.

\section{Discussion And Conclusions}

The diagnosis of GCT-ST based on the preoperative US and CT can be challenging due non-specific manifestations, and difficulty in defining the extent of the tumor. In our study, we used a conventional MRI and DWI approach to investigate two cases of soft tissue giant cell tumor in the limb. The radiologic abnormalities of all lesions were limited to soft tissue without obvious calcification or bone erosion, which are frequently observed at the periphery of the GCT-ST tumors ${ }^{[2-7]}$. In addition, all lesions on MRI 
presented with solid masses, and blurry boundaries on the upper and lower edges. There was a slight hyperintensive signal on $\mathrm{T}_{2}$-weighted images, which indicated that the tumor invaded the neighboring muscles. Thus, our data suggest that MRI-DWI can be useful to identify soft tissue mass with low malignant potential.

In our study, all lesions showed non-homogeneous iso-hypo signal on $\mathrm{T}_{1^{-}}$and iso-hyper signal on $\mathrm{T}_{2^{-}}$ weighted images. In previous reports, Aoki et $a^{\left[{ }^{[3]}\right.}$ and Murphey et $a^{\left[{ }^{[4]}\right.}$ have indicated that MR images of GCT of bone may suggest a presence of solid components with hypointensity to isointensity for $\mathrm{T}_{1}$ - and $\mathrm{T}_{2}$-weighted images, caused by hemosiderin deposition or high collagen content, which is consistent with our findings. On contrast images, the solid regions are intensely slowly enhanced, reflecting the hypervascular tissue in accordance with IHC performance (like the bone GCT), and are in line with findings in other benign soft tissue tumors ${ }^{[2-4]}$, unlike the GCT-ST tumors with diffuse cystic components located in the subcutaneous tissue or malignant soft tissue tumors ${ }^{[8-11]}$.

In the present study, all lesions were significantly big (maximum lesion size ranged from $4.0 \mathrm{~cm}$ to $6.0 \mathrm{~cm}$ ) and were located in the superficial areas of extremities or trunk. This type of lesion is commonly observed in GCT-ST, but it can also be found in the deep soft tissues of head, neck and retroperitomeum region [210]. Unlike deep-seated masses, size criteria (i.e. $5 \mathrm{~cm}$ in diameter) is not an important indicative factor of malignancy for superficial masses ${ }^{[12]}$. To determine the benign characteristic of GCT-ST on MRI, we attempted to evaluate the utility of adding DWI to conventional MRI.

DWI is new method used to differentiate benign from malignant soft tissue lesions based on ADC values; quantitative DWI are useful for differentiating between malignant and benign superficial masses ${ }^{[12-14]}$. In this study, $500 \mathrm{~s} / \mathrm{mm}^{2}$ of $\mathrm{b}$ value was performed and mean ADC value of solid GCT-ST lesions was $2.19 \times 10^{-3} \mathrm{~mm}^{2} / \mathrm{s}$, which was higher compared to the surrounding normal soft tissue. Some authors have reported considerable overlap between the ADC values in benign and malignant tumors, which is thought to be due to the heterogeneous nature of soft-tissue tumors, including intratumoral water content that increases diffusion, and mucinous contents and intratumoral necrosis ${ }^{[12,13]}$. We speculate that high ADC value of solid GCT-ST are more useful in representing tumor histology for intensely enhancing solid masses with no detectable macroscopic necrotic or myxoid predominant areas, although tumors interstitial hemorrhage were detected in the current study. Future studies with a higher sample size are needed to further investigate whether high ADC value is a characteristic of GCT-ST.

Clinically, primary GCT-ST mainly affects young to middle-age adults ${ }^{[4-11,16]}$. Here we reported two cases with GCT-ST: the first was an elder man who was asymptomatic and had a benign clinical course; the other one was a young woman who presented with ill-circumscribed multinodular mass covered by a fleshy red-brown surface and pain in upper limb. Although all upper limb lesions had axillary lymphadenopathy and infiltrated adjacent anatomical structures, including neurovascular bundles, all lesions were unilateral and were identified as benign GCT-ST. 
A noncalcified or nonossified soft-tissue mass with low signal on $\mathrm{T}_{2}$-weighted images is usually fibrous with little cellularity. Since the observed masses with solid component in our study were not bone lesions, Giant cell tumors of tendon sheath (GCT-TS) and giant cell-rich forms of nodular fasciitis (NF) were included in the differential diagnosis. GCT-TS is generally located near joint spaces, while cystic lesion and metaplastic bone formation are absent, and calcification or ossification are very rare. The degree of contrast enhancement of GCT-TS is always higher ${ }^{[17,18]}$. NF is usually located in subcutaneous tissue and it shows hyperintensity on $\mathrm{T}_{2}$-weighted images caused by fluid-filled mucoid spaces. The MRI appearance of the NF is more variable. Emphasis is placed on the differential diagnosis of these giant cell-rich soft tissue neoplasms because clinical behavior, prognosis and treatment can significantly differ [15-22].

In conclusion, our data suggest that DWI could help differentiate benign soft tissue mass compared with other giant cell-rich soft tissue neoplasms or malignant tumors, thus providing more radiologicpathologic information for diagnosis of GCT-ST. The comprehensive imaging of primary GCT-ST can aid to complete tumor resection, which in turn might promote the long survival after surgery.

\section{Abbreviations}

GCT-ST: Soft Tissue Giant Cell Tumor; US: Ultrasonography; CT: Computed Tomography; MRI: Magnetic Resonance Imaging; FSE: Fast spin-echo; FSPGR: Spoiled Gradient Echo Fat Saturation; DWI: Diffusion weighted imaging; IHC: Immunohistochemistry; GCT-TS: Giant cell tumors of tendon sheath

\section{Declarations}

\section{Acknowledgements}

Not applicable

\section{Availability of data and materials}

The datasets used and/or analyzed during the current study are available from the corresponding author on request.

\section{Funding}

The National Natural Science Foundation of China (No. 81771804) and "Basic Scientific Research Projects of the Universities in Liaoning Province (LQ2017013)" supported this work. Lina Zhang is the study project leader; she conceived and participated in the study design and coordination.

\section{Ethics approval and consent to participate}

Institutional review board/ethics committee approval was obtained from the Institutional Review Board of the First Affiliated Hospital of Dalian Medical University (Dalian, China), and was performed in 
accordance with the ethical guidelines of the Declaration of Helsinki. Animals were not used in this study. The patients gave consent to the submission of this case report.

\section{Consent for publication}

Written informed consent was obtained from patients for publication of this study and any accompanying images. A copy of the written consent is available for review by the Editor of this journal.

\section{Competing interests}

The authors declare that they have no competing interests.

\section{Author's contributions}

LZ and HW conceived and participated in study design and coordination, and contributed equally to this work. Jianyun $\mathrm{K}$ and $\mathrm{KZ}$ drafted the manuscript, collected the clinical materials and radiological data, and contributed equally to this work. HW participated in the pathological workup. AL and TL helped to draft the manuscript. All authors read and approved the final manuscript.

\section{Author details}

${ }^{1}$ Department of Radiology, First Affiliated Hospital of Dalian Medical University, No 222 zhongshan Road, Xigang district, Dalian, Liaoning, 116011, China

2Department of Radiology, Second Affiliated Hospital of Dalian Medical University, 467 Zhongshan Road, Shahekou district, Dalian, Liaoning, 116023, China

${ }^{3}$ Department of Pathology, First Affiliated Hospital of Dalian Medical University, No 222 zhongshan Road, Xigang district, Dalian, Liaoning, 116011, China

${ }^{4}$ College of Medical Imaging of Dalian Medical University,No 9 West Section, Lvshun South Road, Dalian, Liaoning, 116044, China

\section{References}

1. Jo VY, Fletcher CD. WHO classification of soft tissue tumours: an update based on the 2013 (4th) edition. Pathology. 2014; 46(2) : 95-104.

2. Lee MY, Jee WH, Jung CK, et al. Giant cell tumor of soft tissue: a case report with emphasis on MR imaging.Skeletal Radiol.2015;44(7):1039-43.

3. Aoki J, Moriya K, Yamashita K, et al. Giant cell tumors of bone containing large amounts of hemosiderin: MR-pathologic correlation. J Comput Assist Tomogr. 1991;15:1024-7.

4. Murphey MD, Nomikos GC, Flemming DJ, et al.From the archives of AFIP. Imaging of giant cell tumor and giant cell reparative granuloma of bone: radiologic-pathologic correlation. Radiographics. 
2001;21:1283-309.

5. Grabellus F, Sheu SY, Scmidt B, et al.Giant cell tumors of soft tissue arising in surgical scars. 2009;30(5):401-6.

6. Sonmez Ergun S, Buyukbabani N, Atilganoglu, U. Primary giant cell tumor of soft tissue mimicking a vascularneoplasm.Dermatol Surg.2008;34(1):102-4.

7. Call $\mathrm{AO}$, Tunakan $\mathrm{M}$, Katilmis $\mathrm{H}$, et al.Soft tissue giant cell tumor of low malignant potential of the neck: a case report and review of the literature.Turk Patoloji Derg.2014;30(1):73-7.

8. Shinji Kishi, Hiroyuki Monma, Hiroshige Hori,et al.First case report of a huge giant cell tumor of soft tissue originating from the retroperitoneum. Am J Case Rep. 2018; 19: 642-50.

9. Sang Bu An, Jung-Ah Choi, Jin-Haeng Chung, et al.Giant cell tumor of soft tissue: a case with atypical US and MRI findings.Korean J Radiol. 2008; 9(5): 462-5.

10. Boneschi V, Parafioriti A, Armiraglio E, et al. Primary giant cell tumor of soft tissue of the groin-a case of 46 years duration.J Cutan Pathol. 2009;36 (Suppl. 1): 20-4.

11. Dodd LG, Major N, Brigman B.Malignant giant cell tumor of soft parts. Skeletal Radiol.2004 ;33(5):295-9.

12. Jeon JY, Chung HW, Lee MH, et al. Usefulness of diffusion-weighted MR imaging for differentiating between benign and malignant superficial soft tissue tumours and tumour-like lesions. $\mathrm{Br} \mathrm{J}$ Radiol 2016; 89: 20150929.

13. Choi, YJ, Lee, IS, Song, YS, et al. Diagnostic performance of diffusion-weighted (DWI) and dynamic contrast-enhanced (DCE) MRI for the differentiation of benign from malignant soft-tissue tumors. Journal of Magnetic Resonance Imaging.2019;50(3):798-809.

14. Bonarelli C, Teixeira PA, Hossu G, et al. Impact of roi positioning and lesion morphology on apparent diffusion coefficient analysis for the differentiation between benign and malignant nonfatty softtissue lesions.AJR Am J Roentgenol. 2015;205(1):W106-13.

15. Khoo MMY, Tyler PA, Saifuddin A,et al. Diffusion-weighted imaging (DWI) in musculoskeletal MRI: A critical review. Skeletal Radiol 2011;40:665-681.

16. Meana Moris AR, García Gonzalez P, Fuente Martin E,et al. Primary giant cell tumor of soft tissue: fluid-fluid levels at MRI(2010:3b).Eur Radiol.2010;20 (6):1539-43.

17. Hourani R, Taslakian B, Shabb NS, et al. Fibroblastic and myofibroblastic tumors of the head and neck: comprehensive imaging based review with pathologic correlation. Eur J Radiol. 2015;84(2): 250-60.

18. Kwak HS, Lee SY, Kim JR,et al.MR imaging of calcifying aponeurotic fibroma of the thigh. Pediatr Radiol.2004;34(5):438-40.

19. Wang C, Song RR, Kuang PD, et al. Giant cell tumor of the tendon sheath: Magnetic resonance imaging findings in 38 patients. Oncol Lett.2017;13(6):4459-4462.

20. Christina M Ward, Nathan E Lueck, Curtis M Steyers.Acute Carpal Tunnel Syndrome Caused by Diffuse Giant Cell Tumor of Tendon Sheath: A Case Report. lowa Orthop J. 2007; 27: 99-103. 
21. Wang XL, De Schepper AM, Vanhoenacker F, et al. Nodular fasciitis: correlation of MRI findings and histopathology.Skeletal Radiol.2002;31(3):155-61

22. Nakayama R, Jagannathan JP, Ramaiya N, et al. Clinical characteristics and treatment outcomes in six cases of malignant tenosynovial giant cell tumor: initial experience of molecularly targeted therapy.BMC Cancer.2018;18(1): 1296.

\section{Figures}
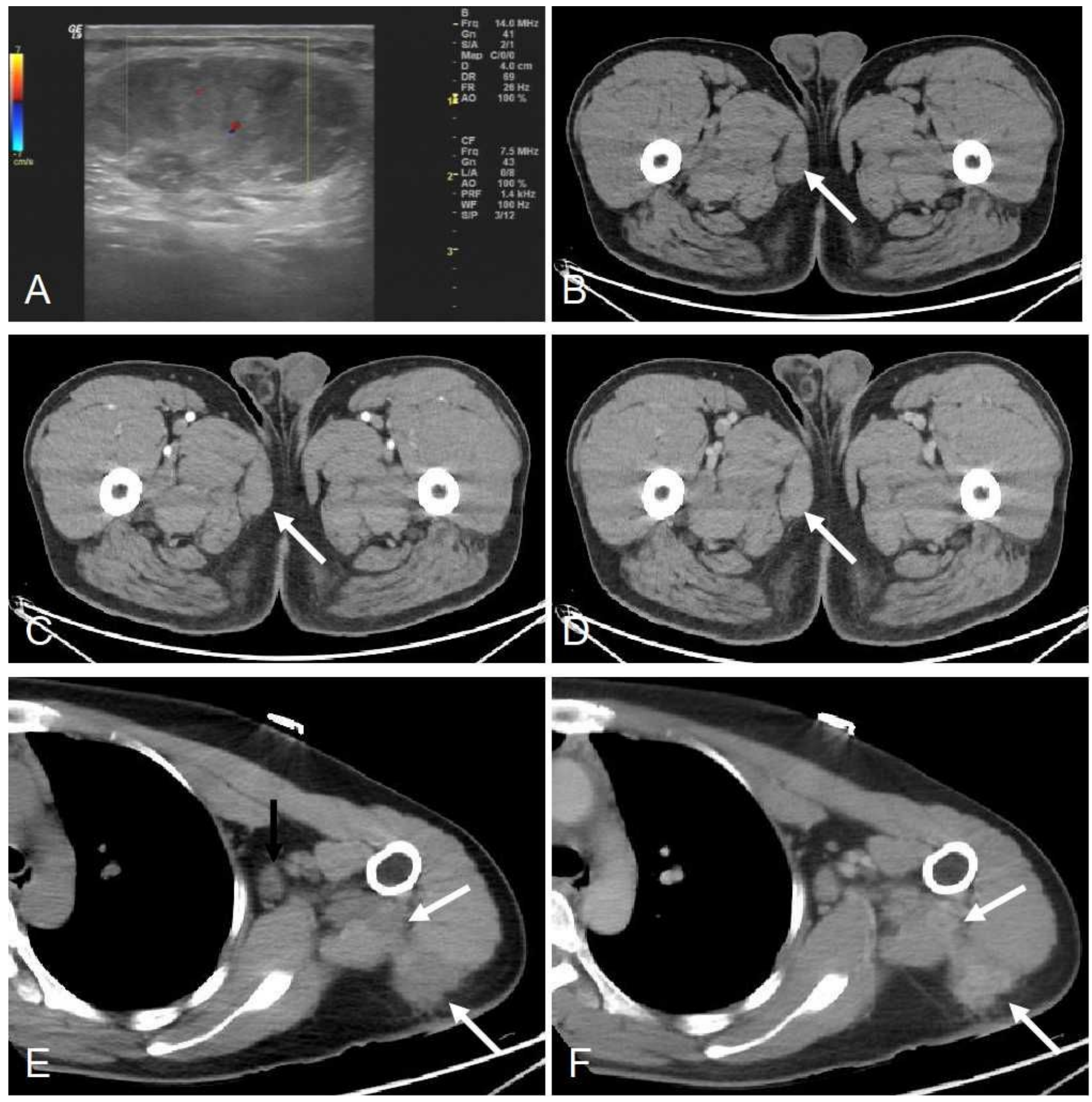


\section{Figure 1}

(a-d) Case 1, 60-year-old man with giant cell tumor of soft tissue in right thigh. a The Doppler sonographic examination shows a few vascular signals within the well-circumscribed large solid mass. $b$ Computed tomography imaging shows an isodensity mass with enlargement of the right gracilis muscle (white arrow). c-d Contrast CT reveals a slightly persistent homogeneous enhancement of the mass with a blurred margin (white arrow). (e-f) Case2, 36-year-old woman with giant cell tumor of soft tissue in right upper limb. e Plain CT imaging shows a irregular hypodensity mass in the superficial deltoid muscle extending to the intermuscular space (white arrow) with axillary lymphadenopathy (black arrow). $f$ Contrast CT(venous phase) reveals a slightly persistent inhomogeneous enhancement of the mass with a blurred margin (white arrow).
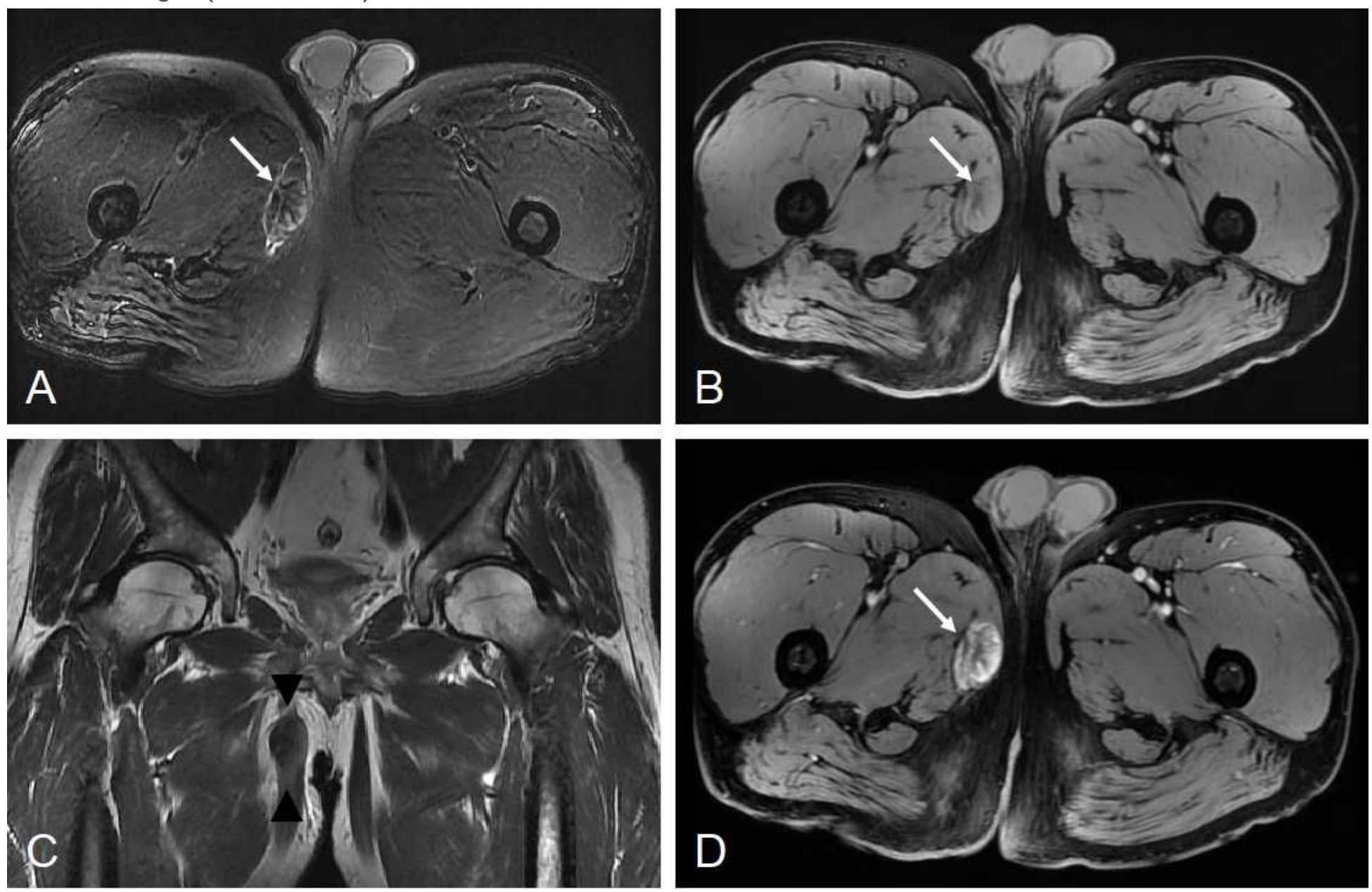

Figure 2

MR imaging manifestations of the tumors. a Axial T2-weighted with fat saturation image shows a solid mass with intermediate signal intensity (white arrow) in comparison to muscle intensity. b Axial T1 FSPGR (pre-contrast enhancement) shows a hypo-iso intensity signal mass (white arrow).c Coronal T2weighted image shows solid mass with hypo-iso signal intensity (white arrow) relative to muscle. A welldefined margin adjacent to the subcutaneous tissue, while the upper and lower edges of the mass were 
blurred with a slightly hyperintense signal (black triangle). d Axial T1 FSPGR (with contrast enhancement) shows heterogeneously intense enhancement of the mass.
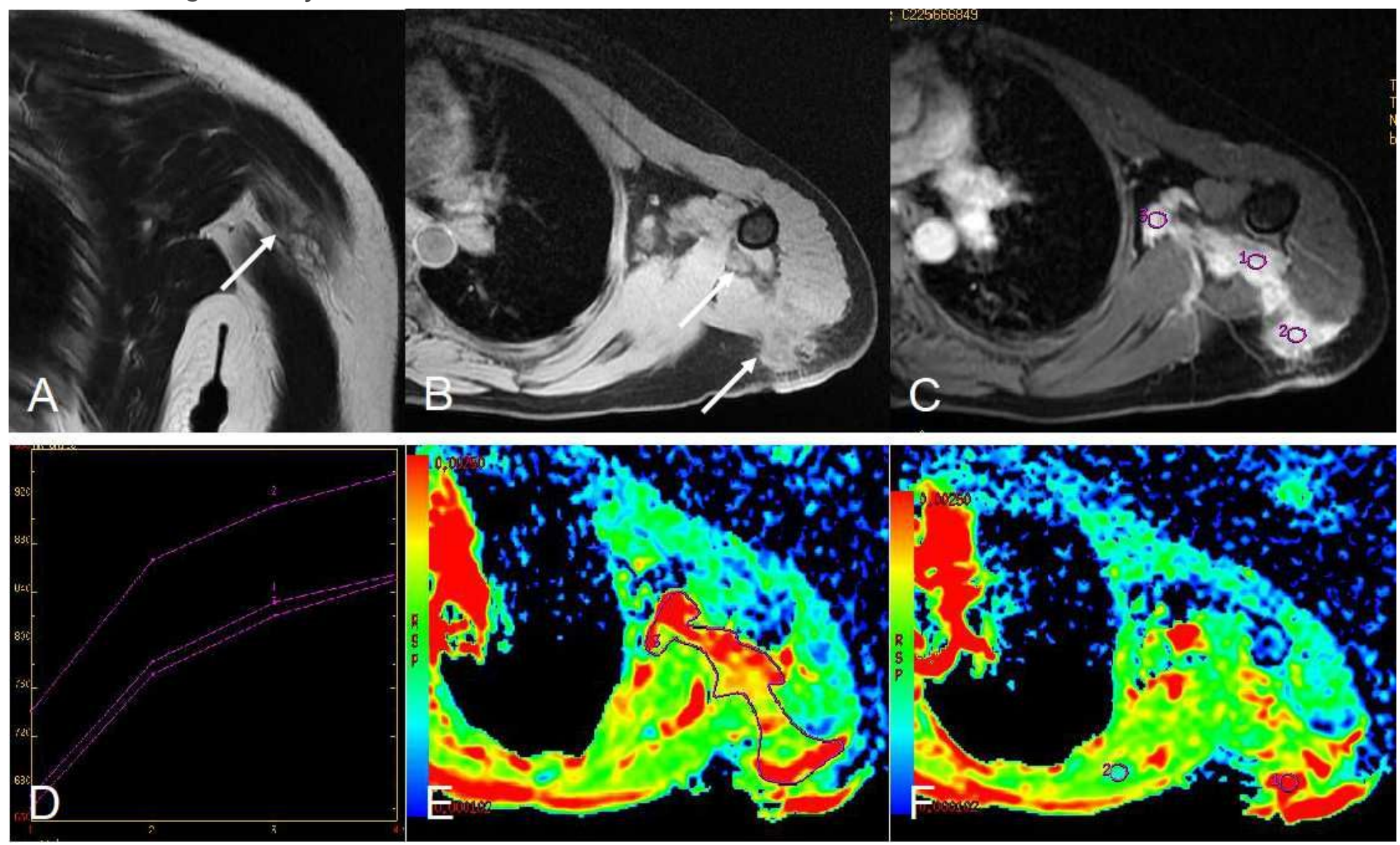

\section{Figure 3}

Conventional MRI combined with diffusion weighted MRI. a Coronal T2-weighted without fat saturation image shows iso-hyper signal intensities(white arrow) in comparison to muscle intensity.b Axial T1 FSPGR (pre-contrast enhancement) shows a hypo-iso intensity signal mass (white arrow), relative to muscle signal intensity. c Axial dynamic 3D T1 FSPGR (Lava) sequence shows multiple nodular enhancements with irregular shapes, and margins were blur(ROI1, 2, 3). d TIC appeared with a slow increase type. e Lesions on DWI ( $b=500 \mathrm{~s} / \mathrm{mm} 2)$ were hyperintense with an higher mean ADC value of $2.19 \times 10-3 \mathrm{~mm} 2 / \mathrm{s}$ (ROI 3)than surrounding normal soft tissue (1.03× 10-3 mm2/s) (f, ROI2). 

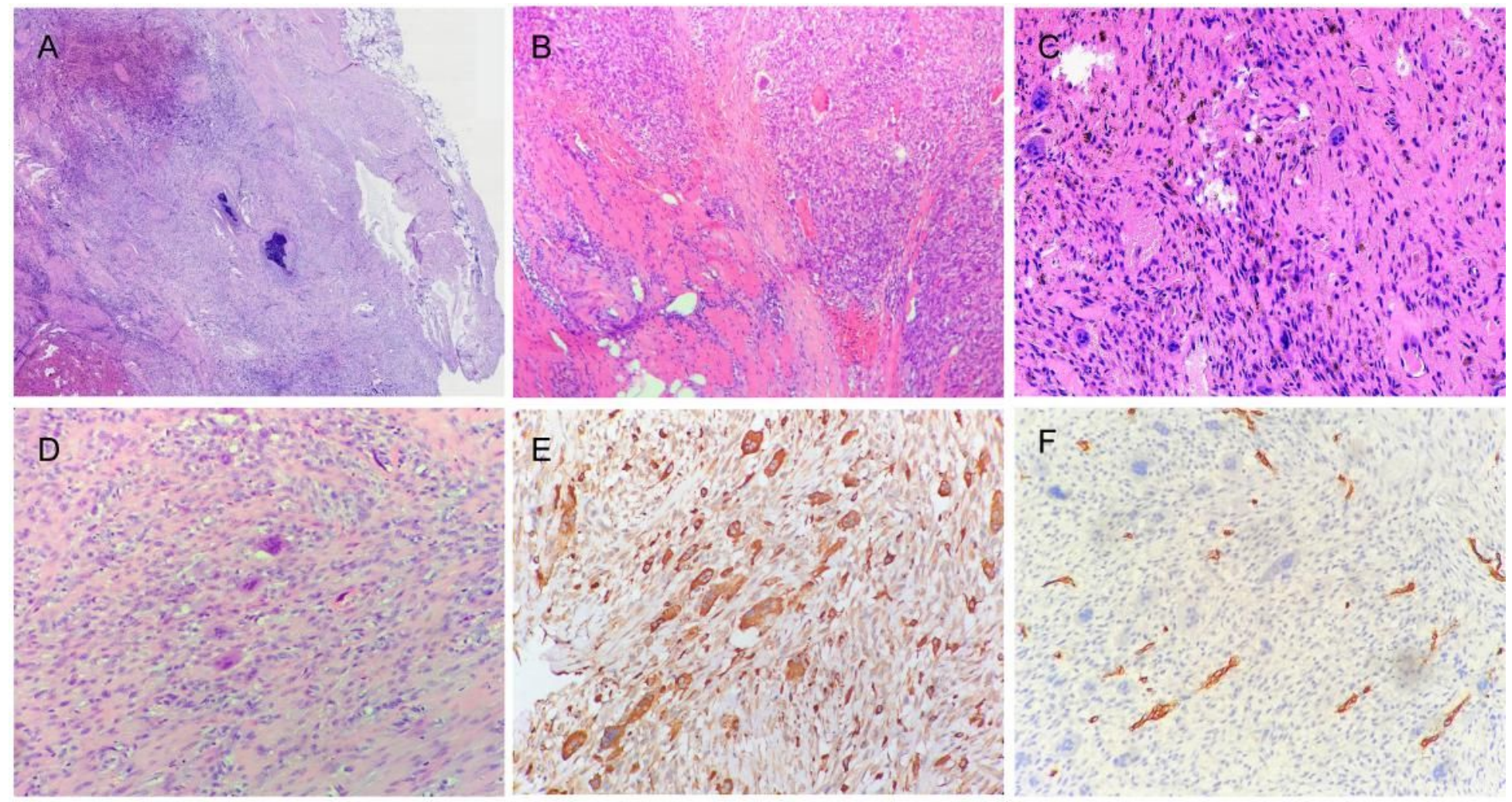

\section{Figure 4}

Pathological features of the tumor. a The relatively clear boundary of the tumor. b The tumor had invaded into the neighboring muscles on one side. $\mathrm{C}$ Hemosiderin-containing cells indicated a tumor interstitial hemorrhage. $d$ The tumor had many multinucleated giant cells, surrounded by spindle cells. e CD68 on multinuclear cells was strongly stained. f CD34 was not stained in the tumor cells but was reactive to the vascular endothelial cells in the stroma. 